\title{
Why the immune system takes its chances with randomness
}

\author{
Philip D. Hodgkin, Mark R. Dowling and Ken R. Duffy
}

In their recent Opinion article (Lymphocyte fate specification as a deterministic but highly plastic process. Nature Rev. Immunol. 14, 699-704 (2014)) $)^{1}$, Reiner and Adams presented a fascinating deterministic interpretation of how lymphocytes acquire different fates. They propose that the generation of multiple lymphocyte subsets from each precursor occurs via an inevitable developmental pathway. This deduction is based on the premise that the system is too important to be left to stochastic processes. To account for recent evidence to the contrary, stochastic processes are suggested to only appear under conditions in which artificially large numbers of responding precursors might relax the deterministic programme (as used in REFS 2,3) or under in vitro conditions in which the usual three-dimensional (3D) arrangement of externally delivered signals that channel fates is removed (as used in REF. 4). In other words, stochastic mechanisms only occur when experimental conditions happen to support the role of randomness.

There are, however, several reasons - as outlined below - to challenge the premise that stochastic processes are not equally up to the task of generating a reliable immune response.

\section{Precedent}

The authors themselves point out that evolution exploits randomness for the most important task of all - creating lymphocyte receptor diversity. Other immune examples of stochastic processes include the probabilistic expression of cytokines ${ }^{5,6}$ and the combinatorial expression of natural killer cell receptors in a population?

\section{Efficiency}

In the imagined B cell and T cell odysseys ${ }^{1}$, at least six intricate moves must take place to generate the different cell fates. A distinct deterministic pathway is needed for each, and the correct set of signals must be received in the correct order by each of potentially thousands of progeny; lymphocytes and numerous other cells must encode complex instructions for orchestrating the right set of signals to generate every cell type at the right time. By contrast, by using stochastic processes multiple cell types can be generated with much simpler instructions ${ }^{4,8-11}$, even in the absence of environmental direction.

\section{Reductionism}

It is tempting to observe the complex structures and cell interactions of primary lymphoid tissue and deduce that they are crucial for the formation of heterogeneous outcomes. This hypothesis has been tested by asking what remains when such structures are removed. We and others find a great deal of cell fate heterogeneity under simple in vitro culture conditions ${ }^{4-6,12,13}$. Conversely, crucial molecular contributors to early developmental programmes, including asymmetric cell division, do not alter $\mathrm{B}$ cell or $\mathrm{T}$ cell responses in vivo ${ }^{14}$. Thus, although the 3D environment and asymmetric programming might have some role in modifying cell fate allocation, they are not the only sources of variation.

\section{Extrapolation}

In the stochastic interpretation, variation is inherent and consistent immune outcomes only arise when considering the population as a whole. As Reiner and Adams point out, the number of antigen-specific precursors recruited into the immune response is a crucial variable, and may be as low as 20 . However, mathematical models in which randomness drives cell fate selection suggest that a reasonably robust immune response can be achieved even with starting cell numbers of this order ${ }^{4,9,10,15}$. Thus, a role for randomness should not be rejected on this basis alone.

\section{Summary}

As a research community, we have not yet acquired all of the data required to answer how both deterministic and stochastic processes interleave to build the complete immune response. However, along with Reiner and Adams, we look forward to the resolution of this conundrum. Perhaps unlike them, however, we are gamblers, suspecting that the immune system does play a game of chance, albeit with the rules having evolved so that the odds are stacked in our favour.

Philip D. Hodgkin and Mark R. Dowling are at the Walter and Eliza Hall Institute of Medical Research, Parkville, Melbourne, 3052 Victoria, Australia, and the Department of Medical Biology, University of Melbourne, Melbourne, 3010 Victoria, Australia. Ken R. Duffy is at the Hamilton Institute, National University of Ireland, Maynooth, County Kildare, Ireland.

Correspondence to P.D.H e-mail:hodgkin@wehi.edu.au doi:10.1038/nri3734-c1

1. Reiner, S. L. \& Adams, W. C. Lymphocyte fate specification as a deterministic but highly plastic process. Nature Rev. Immunol. 14, 699-704 (2014).

2. Buchholz, V. R. et al. Disparate individual fates compose robust $\mathrm{CD} 8^{+} \mathrm{T}$ cell immunity. Science 340, 630-635 (2013).

3. Gerlach, C. et al. Heterogeneous differentiation patterns of individual CD8 ${ }^{+} \mathrm{T}$ cells. Science 340 , 635-639 (2013).

4. Duffy, K. R. et al. Activation-induced B cell fates are selected by intracellular stochastic competition. Science 335, 338-341 (2012).

5. Guo, L., Hu-Li, J. \& Paul, W. E. Probabilistic regulation in $\mathrm{T}_{\mathrm{H}} 2$ cells accounts for monoallelic expression of IL-4 and IL-13. Immunity 23, 89-99 (2005).

6. Kelso, A., Groves, P., Troutt, A. B. \& Francis, K. Evidence for the stochastic acquisition of cytokine profile by $\mathrm{CD} 4^{+} \mathrm{T}$ cells activated in a T helper type 2 -like response in vivo. Eur. J. Immunol. 25, 1168-1175 (1995)

7. Raulet, D. H. et al. Specificity, tolerance and developmental regulation of natural killer cells defined by expression of class I-specific Ly 49 receptors. Immunol. Rev. 155, 41-52 (1997).

8. Rohr, J. C., Gerlach, C., Kok, L. \& Schumacher, T. N Single cell behavior in T cell differentiation. Trends Immunol. 35, 170-177 (2014).

9. Duffy, K. R. \& Hodgkin, P. D. Intracellular competition for fates in the immune system. Trends Cell Biol. 22, 457-464 (2012)

10. Subramanian, V. G., Duffy, K. R., Turner, M. L. \& Hodgkin, P. D. Determining the expected variability of immune responses using the cyton model. J. Math. Biol. 56, 861-892 (2008).

11. Hodgkin, P. D. A probabilistic view of immunology: drawing parallels with physics. Immunol. Cell Biol. 85, 295-299 (2007).

12. Hasbold, J., Corcoran, L. M., Tarlinton, D. M., Tangye, S. G. \& Hodgkin, P. D. Evidence from the generation of immunoglobulin G-secreting cells that stochastic mechanisms regulate lymphocyte differentiation. Nature Immunol. 5, 55-63 (2004).

13. Bird, J. J. et al. Helper T cell differentiation is controlled by the cell cycle. Immunity 9, 229-237 (1998).

14. Hawkins, E. D et al Regulation of asymmetric cell division and polarity by Scribble is not required for humoral immunity. Nature Commun. 4, 1801 (2013).

15. Duffy, K. R. \& Subramanian, V. G. On the impact of correlation between collaterally consanguineous cells on lymphocyte population dynamics. J. Math. Biol. 59, 255-285 (2009)

\section{Acknowledgements}

P.D.H. and M.R.D. are supported by National Health and Medical Research Council (NHMRC) Fellowships and NHMRC grants 1057831 and 1054925, and Independent Research Institutes Infrastructure Support Scheme Grant 361646. K.R.D. is supported by Science Foundation Ireland grant 12 IP 1263. P.D.H. and K.R.D. are also supported by the Human Frontier Science Program, grant RGP0060/2012.

\section{Competing interests statement}

The authors declare no competing interests. 